\title{
The value of MRI in evaluation of shoulder pain
}

\author{
Safaa Aboelkaseem Mohamed ${ }^{1}$, Osama Mohamed Ebied ${ }^{1}$, Mohamed Shawky abdullah ${ }^{1}$, \\ Hala Hafez Mohamed ${ }^{1}$, Hesham Mohamed Zaky EImowafy ${ }^{2}$ \\ ${ }^{1}$ Radiology department Faculty of Medicine, Menoufia University, Shbine Elkoom, Egypt \\ ${ }^{2}$ Orthopedic department, Faculty of Medicine, Menoufia University, Shbine Elkoom, Egypt
}

\section{Email address:}

hefezhala@yahoo.com (H. H. Mohamed), Osamaebied@hotmail.com (O. M. Ebied), M.shawkey@yahoo.com (M. S. Abdullah), helmowafy@hotmail.com (H. M. El-mowafy),Safaa_aboelkasem@yahoo.com (S. A. M. Soliman)

\section{To cite this article:}

Safaa Aboelkaseem Mohamed, Osama Mohamed Ebied, Mohamed Shawky abdullah, Hala Hafez Mohamed, Hesham Mohamed Zaky Elmowafy. The Value of MRI in Evaluation of Shoulder Pain. International Journal of Medical Imaging. Vol. 2, No. 4, 2014 , pp. 83-91. doi: 10.11648/j.ijmi.20140204.11

\begin{abstract}
Objectives: To evaluate the role of MRI, MR Arthrography compared to arthroscopy in shoulder pain. Methods: This study was conducted on 100 patients complaining of shoulder pain. The patients were referred to the radiology department from the out patient clinics and the orthopedics department of Menoufia University Hospital. All patients were examined by conventional MRI while only 8 of them had additional MRA. Arthroscopy was performed to all patients. Results: There were fifty six males and forty four females, their ages ranged between $19 \& 69$ years (mean age, 31 years). There is significant positive correlation between MRI and arthroscopy with $r=+0.9,95 \%$ CI $(0.82-0.99)$ and $p$ value $=0.003$. MRI showed $100 \%$ specificity for anterior labral tears, SS (supraspinatus) partial thickness tear and SS full thickness tear and $98.9 \%$ specificity for SLAP (superior labrum anterior to posterior). MRI showed $76.9 \%$ sensitivity for anterior labral tears and SS partial thickness tear. Conventional MRI showed 54.5\% sensitivity in diagnosis of SLAP tears and $83.3 \%$ sensitivity in diagnosis of supraspinatous full thickness tears. Conclusion: MRI of the shoulder is an accurate, useful and established technique for assessment of different shoulder lesions.
\end{abstract}

Keywords: Magnetic Resonance Imaging, Magnetic Resonance Arthrography, Shoulder Pain

\section{Introduction}

Shoulder pain is a common complaint by orthopedic patients, and it can be due to a variety of causes. Magnetic resonance imaging (MRI) has played an increasingly important role as a noninvasive test for accurate diagnosis of various causes of shoulder pain and determining which patients may benefit from surgery (1).

The shoulder is the most mobile and therefore the most unstable of all the joints of the human body. Repetitive active and passive forces render the tendons that form the rotator cuff susceptible to degeneration leading to swelling of the tendon and a minor degree of subluxation (2).

Imaging plays a major role in the management of shoulder problems. MRI \& MRA have replaced arthrography for evaluating the integrity of the rotator cuff and shoulder instability. The shoulder is commonly imaged using MRI, with or without intraarticular contrast medium. Some anatomic structures, such as the rotator cuff tendons and bony components, can be assessed without arthrographic technique, whereas the glenohumeral ligaments and labrum require arthrographic technique for more accurate assessment (3).

Patients presenting for imaging fall broadly into one of the following categories: non specific pain, pain and restricted movement on abducting the arm (implying impingement), and symptoms of instability. Non-specific pain may be due a range of conditions such as arthropathies, tumour and infection (4).

In the workup of patients with shoulder pain, the role of imaging is to determine the cause, guide treatment decisions and furthermore, the surgical approach, open versus arthroscopic, can be chosen once the correct diagnosis is made (5).

\section{Patients and Methods}

\subsection{Patients}

This study was performed between April 2011 and September 2013 and was conducted on 100 patients 
complaining of shoulder pain. There were fifty six males and forty four females, their ages ranged between $19 \& 69$ years (mean age, 31 years). The patients were referred to the radiology department from the out patient clinics and the orthopedic department of Menoufia University Hospital for MRI examination of the shoulder.

\subsection{Conventional MRI}

The MRI machine used was Toshiba Exclart Vantage 1.5-T scanner. The examination protocol included oblique coronal, oblique sagittal, and axial planes. Oblique coronal and sagittal fast spin-echo T1-weighted (TR/TE, 405/15), oblique coronal fast spin-echo T2-weighted (TR/TE, 4000/80), oblique coronal gradient and axial gradient (TR/TE, 435/15) and oblique coronal STIR (TR/TE, 20000/48) sequences with a field of view of $12-18 \mathrm{~cm}$ were used. Slice thickness was $2.5-4 \mathrm{~mm}$ with a $10 \%$ interslice gap.

Technique of examination:

The MRI machine contains a magnet, thus metalcontaining objects are not allowed into the scanner area. Removable dental works are removed just before the scan. The patients are asked to remove any clothes containing metal objects and to wear a hospital gown.

MRI examination of the shoulder takes 20- 25 minutes, thus the patient lies in a comfortable position so that he or she remains stationary for a fairly long period of time. A single-loop curved surface coil is firmly fitted to the patient's shoulder. A pillow is placed beneath the knees to reduce the stress on the back and pads are placed at pressure points.

The patient lies supine on the MR table with the arm beside the body in a neutral position. A preliminary scout image is taken in the coronal plane to serve as a localizer for subsequent pulse sequences.

The next set of images is acquired in the trans-axial plane perpendicular to the long axis of the glenohumeral articulation on the coronal scout images. It extends from the top of the acromion through the inferior margin of the glenoid.

Coronal oblique images are obtained in a plane parallel to the supraspinatus tendon so that the rotator cuff muscles and tendons can be seen in continuity. The cursors are placed along the axis of the central supraspinatus tendon on the axial images. Sequences in the oblique-coronal plane are obtained from the region in front of the coracoid process through the posterior musculature.

An oblique-sagittal plane is then selected perpendicular to the oblique-coronal images and parallel to the glenoid rim. The field of view should include the acromion, entire humeral head and scapula.

All our patients were examined by conventional MRI while only 8 of them had additional MR arthrography (MRA) study. This was followed by arthroscopy to all patients.

\subsection{Magnetic Resonance Arthrography (MRA)}

As previously mentioned MR arthrography was only performed to 8 patients within one week after the conventional MRI study. The injection of contrast material for arthrography was performed in the ultrasound unit of the radiology department. Hitachi imaging system ultrasound device equipped by a 6-7.5 MHz linear array transducer was used for all patients. An anterior approach was used for all patients. The patient was asked to lie supine and expose the affected shoulder; the arm was extended and externally rotated. The transducer was placed at the same side of the affected shoulder. The transducer was placed ventrally parallel to the long axis of the subscapular tendon. A transverse image of the anterior shoulder joint was obtained by using the coracoid process medially and the anteromedial humeral head laterally as reference points. The joint space should be clearly visualized in this plane. Using anti septic technique and without moving the ultrasound transducer from the desired transverse plane, the needle is advanced into the joint space through the rotator cuff interval. A $4 \mathrm{~cm}$ green needle $(21 \mathrm{G})$ is used. The needle is inserted into the shoulder approximately midway between the two reference points until it makes contact with the medial edge of the upper humeral head, with the beveled edge of the needle facing towards the humeral head. A local anesthetic (lidocaine ampule) is administered as a test-injection, low resistance while injecting confirms the intra-articular position of the needle. If high resistance is felt, the needle can be slightly angled medially away from the bony humeral surface. The administration of local anaesthetic can be watched carefully with the real-time ultrasound. Then, by simply changing syringes, the diluted gadolinium-saline contrast solution ( $0.2 \mathrm{ml}$ gadolinium mixed with $20 \mathrm{ml}$ saline) is injected, while continuously watching on the real-time ultrasound monitor. Retrograde flow from the needle after the syringe was disconnected indicates adequate joint distension.

After injection of this mixture, shoulder exercise is done then the patient is transferred into the MRI unit. T1weighted fat-saturated oblique coronal, oblique sagittal, and axial images are taken. All MR arthrography images are obtained with $12-16 \mathrm{~cm}$ field of view and a 3.5- $4 \mathrm{~mm}$ slice thickness (with a $10 \%$ interslice gap). The rest of the MRI parameters are the same as those for conventional MRI. The images are examined for the presence or absence of different pathological lesions that may explain the cause of shoulder pain.

\subsection{Statistical Analysis}

The sensitivity and specificity of $1.5 \mathrm{~T}$ conventional MRI for detection of labral tears, and full- and partial-thickness supraspinatus tendon tears compared with arthroscopy are calculated. The true- and false-positive and true- and falsenegative findings compared with arthroscopy were calculated. 
Table (1). conventional MRI pathologic findings

\begin{tabular}{ll}
\hline MRI findings & frequency \\
\hline Acromion Shape & Type I =97 \\
& Type II $=0$ \\
& Type III =3 \\
& Type IV $=0$ \\
& 85 \\
Acromio-clavicular osteoarthritis & 9 \\
Subacromial bursitis & 45 \\
SS tendinosis without tears & 30 \\
SS partial thickness tear & 10 \\
SS full thickness tear & 10 \\
Anterior labral tear & 7 \\
SLAP tear & 3 \\
Biceps tenosynovitis & \\
\hline
\end{tabular}

$\mathrm{SS}=$ supraspinatus

$\mathrm{SLAP}=$ superior labrum anterior to posterior
Using Chi-Square Tests (Pearson's Chi-Square, Likelihood Ratio and Linear-by-Linear Association) $\mathrm{P}$ value is considered significant if $<0.01$ and non significant if $\mathrm{P}>0.01$.

Table (2). the frequency of the different pathological findings among the patients in relation to the mean age (31 years)

\begin{tabular}{|c|c|c|c|c|}
\hline Lesions & $<31 Y$ & $\geq 31 Y$ & $\begin{array}{l}\text { Chi square } \\
\text { Test X2 }\end{array}$ & $P$ value \\
\hline $\begin{array}{l}\text { Acromio-clavicular } \\
\text { osteoarthritis }\end{array}$ & 15 & 70 & \multirow{7}{*}{31.77} & \multirow{7}{*}{$<0.0001$} \\
\hline Subacromial bursitis & 3 & 6 & & \\
\hline SS partial thickness tear & 8 & 22 & & \\
\hline SS full thickness tear & 1 & 9 & & \\
\hline Anterior labral tear & 8 & 2 & & \\
\hline SLAP tear & 6 & 1 & & \\
\hline Biceps tenosynovitis & 1 & 2 & & \\
\hline
\end{tabular}

Table 3. Pearson's correlation between MRI findings and diagnostic shoulder arthroscopy results

\begin{tabular}{lllllllll}
\hline & $\begin{array}{l}\text { MRI } \\
\text { results }\end{array}$ & $\begin{array}{l}\text { Arthroscopy } \\
\text { results }\end{array}$ & $\begin{array}{l}\text { True } \\
+\mathbf{v}\end{array}$ & $\begin{array}{l}\text { True }- \\
\text { ve }\end{array}$ & $\begin{array}{l}\text { False }+ \\
\text { ve }\end{array}$ & $\begin{array}{l}\text { False }- \\
\text { ve }\end{array}$ & $\begin{array}{l}\text { Sensitivit } \\
\mathbf{y} \%\end{array}$ & $\begin{array}{l}\text { Specificit } \\
\mathbf{y} \%\end{array}$ \\
\hline Anterior Labral tears & 10 & 13 & 10 & 87 & 0 & 3 & 76.9 & 100 \\
SLAP & 7 & 11 & 6 & 89 & 1 & 5 & 54.5 & 98.9 \\
SS partial thickness tear & 30 & 39 & 30 & 61 & 0 & $95 \%$ CI & 76.9 & 100 \\
SS full thicknes tear & 10 & 12 & 10 & 88 & 0 & 2 & 83.3 & 100 \\
\hline
\end{tabular}

\section{Results}

Seventy nine patients suffered right shoulder pain while twenty one had left side pain ( $\mathrm{P}$ value $<0.0001)$. Acromioclavicular joint osteoarthritis was the most common pathologic finding (85 patients, fig. $5 \mathrm{E}$ straight arrow) followed by supraspinatus tendinosis (45 patients, fig. $4 \mathrm{C}$ broad arrow). Biceps tenosynovitis (3 patients) and SLAP tears ( 7 patients) were the least common (table 1).

Labroligamentous injuries were more frequent among patients below 31 years while rotator cuff tendon injuries

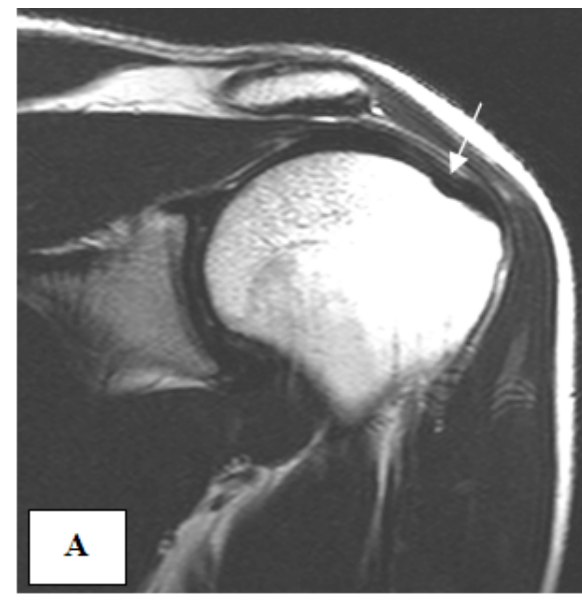

were more common in patients above 31 years (table 2 ).

There was significant positive correlation between MRI and arthroscopy with $\mathrm{r}=+0.9,95 \% \mathrm{CI}(0.82-0.99)$ and $\mathrm{p}$ value $=0.003$ (table 3$)$. MRI showed $100 \%$ specificity for diagnosis of anterior labral tears, supraspinatus partial thickness tear and supraspinatus full thickness tear and $98.9 \%$ specificity for diagnosis of SLAP. It also showed $76.9 \%$ sensitivity for detection of anterior labral tears and supraspinatus partial thickness tear. In diagnosis of SLAP, MRI showed 54.5\% sensitivity and in diagnosis of supraspinatus full thickness tears $83.3 \%$ sensitivity (table 3 ).

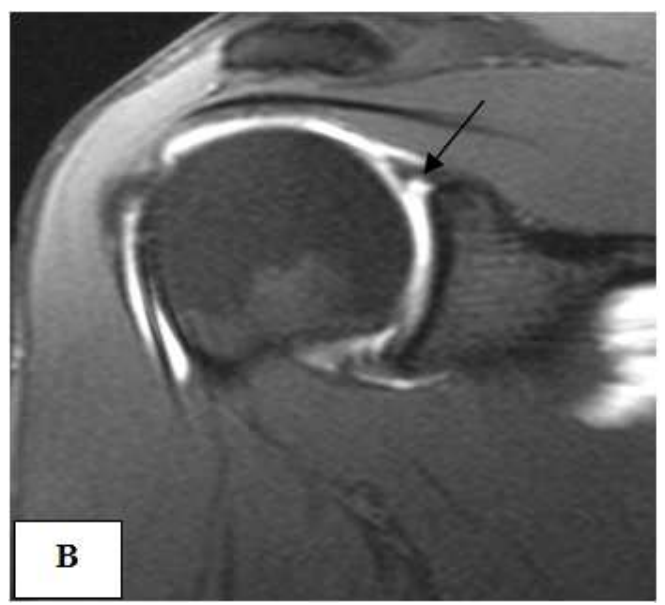



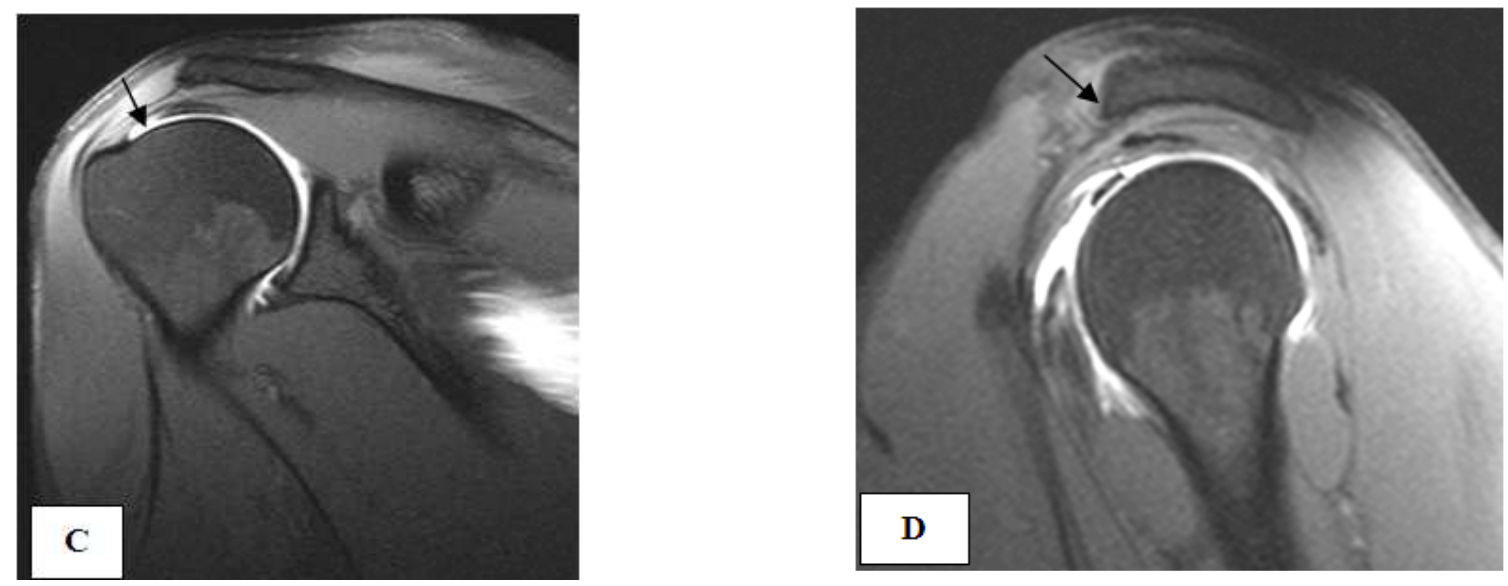

Fig. 1 (A-D). Male patient 33 years old complaining of progressive shoulder pain of 2 years duration Image A (coronal oblique T2WI) shows intermediate signal in the supraspinatus tendon (arrow). Image B (coronal oblique T1WI with fat sat) following arthrography shows a defect at the base of the superior labrum more than $3 \mathrm{~mm}$ in diameter (arrow). Image C (coronal oblique TIWI with fat sat) following arthrography shows a defect at the supraspinatus tendon articular surface (arrow).Image D (sagittal T1WI with fat sat) following arthrography shows acromial spur (arrow).
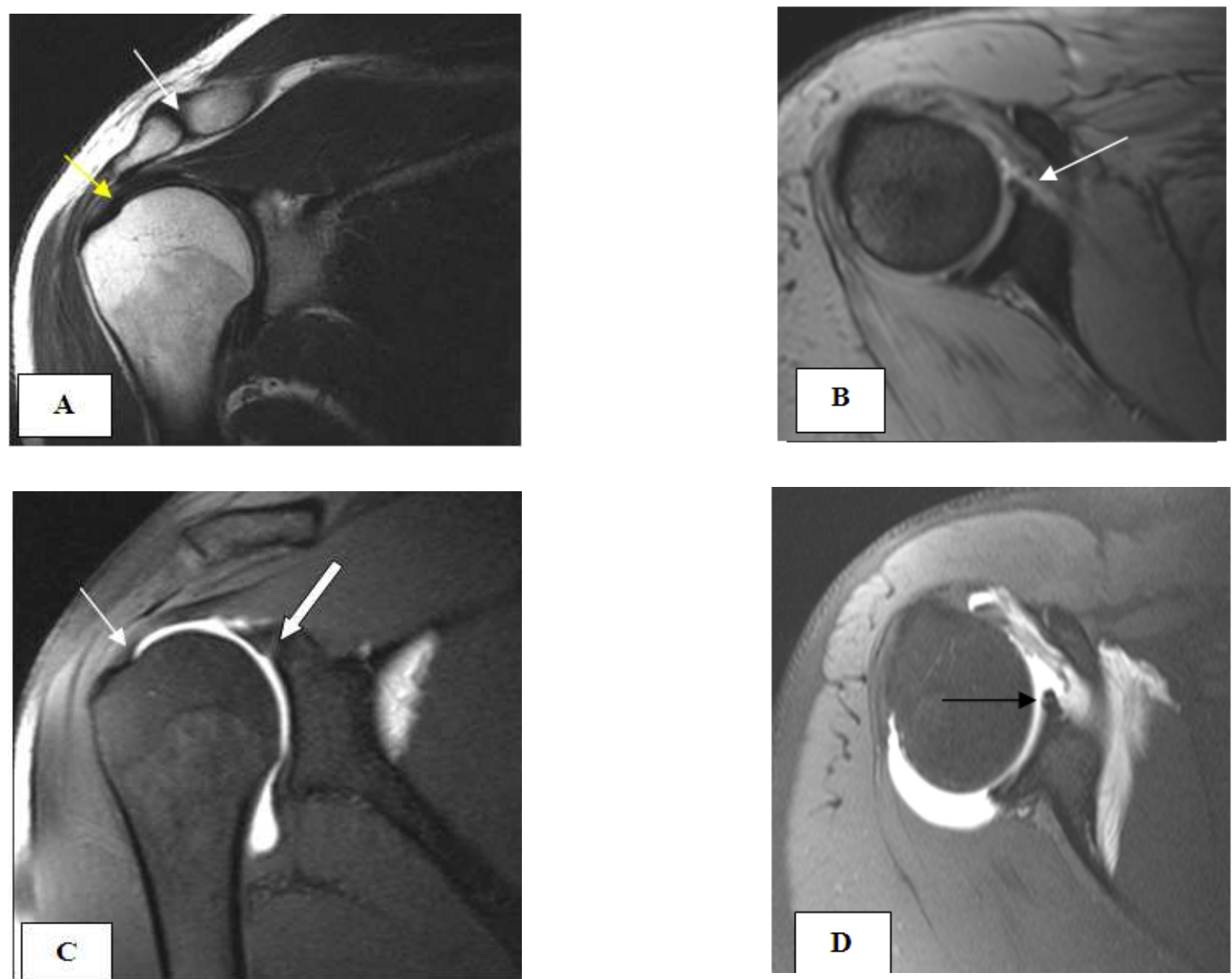

Fig. 2 (A-D). Male patient 31 years old complaining of shoulder pain for 5 months. Image A (coronal oblique T2WI shows intermediate signal in the supraspinatus tendon (yellow arrow) and acromioclavicular joint osteoarthritis (white arrow). Image B (axial gradient) at about 2 o'clock shows defect at the anterosuperior labrum (arrow). Image C (coronal oblique TIWI with fat sat) post arthrography shows intact supraspinatus tendon (narrow long arrow) and intermediate signal inside the superior labrum (broad short arrow).Image D (axial TIWI with fat sat) post arthrography at the same level of (B) shows defect at the anterosuperior labrum (arrow) more evident than in (B). 

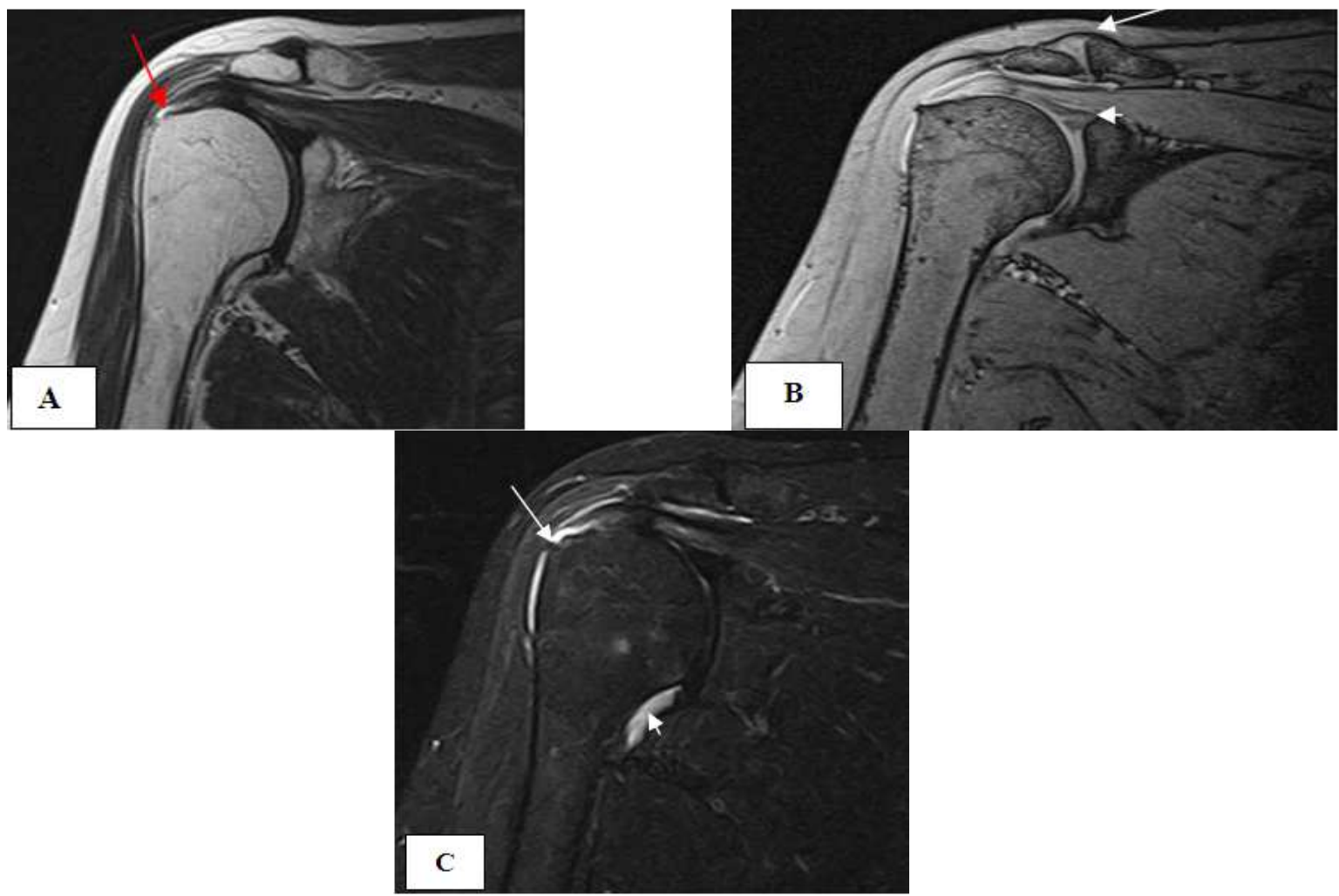

Fig. 3 (A-C). Female patient 52 years old complaining of shoulder pain with limitation of movement for 7 years which became more severe. Image A (coronal oblique T2WI) shows hyper intense signal within the supraspinatus tendon (arrow). Image B: (coronal oblique gradient) shows acromioclavicular osteoarthritis (large arrow) and a defect at the superior labrum dividing it into upper and lower parts with no extension into the biceps tendon (small arrow). Image C (oblique coronal STIR) shows hyperintense signal within the supraspinatus tendon with still intact fibers (large arrow) and mild joint effusion (small arrow).
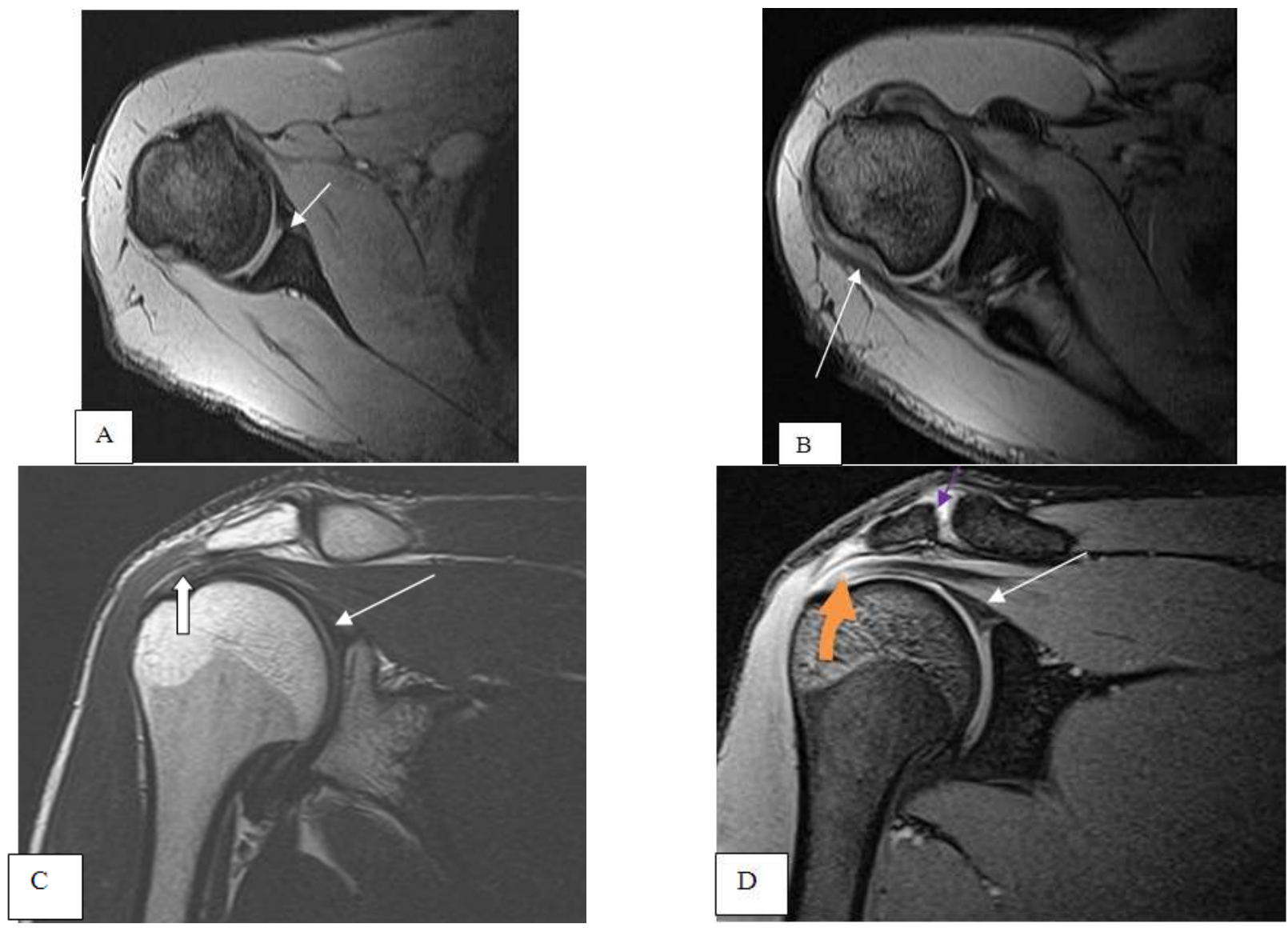


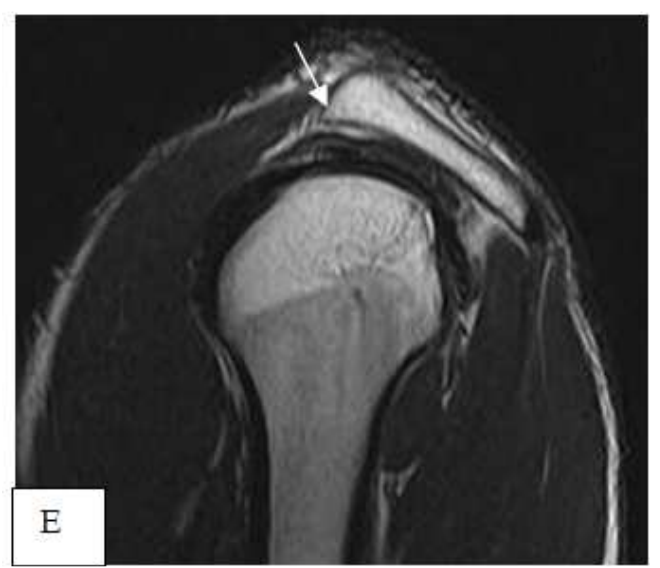

Fig. 4 (A-E). Male patient complaining of shoulder pain of two years duration and one attack of dislocation following direct trauma 4 months ago. Image A (axial gradient) at mid axial level shows detached anterior labrum with a part of the underlying bony glenoid (arrow). Image B (axial gradient) at higher level than (A) shows bone defect at the posterolateral aspect of the humeral head, Hill Sach's fracture (arrow). Image C (oblique coronal T2WI) shows intermediate signal within the superior labrum (large narrow arrow), acromioclavicular osteoarthritis (small narrow arrow) and intermediate signal within the supraspinatus tendon (broad arrow). Image D (oblique coronal gradient) shows acromioclavicular osteoarthritis (purple arrow), intermediate signal within the superior labrum (white arrow) and intermediate signal within the supraspinatus tendon (orange arrow). Image E (sagittal T2WI) shows acromion type I with acromial spur (arrow).
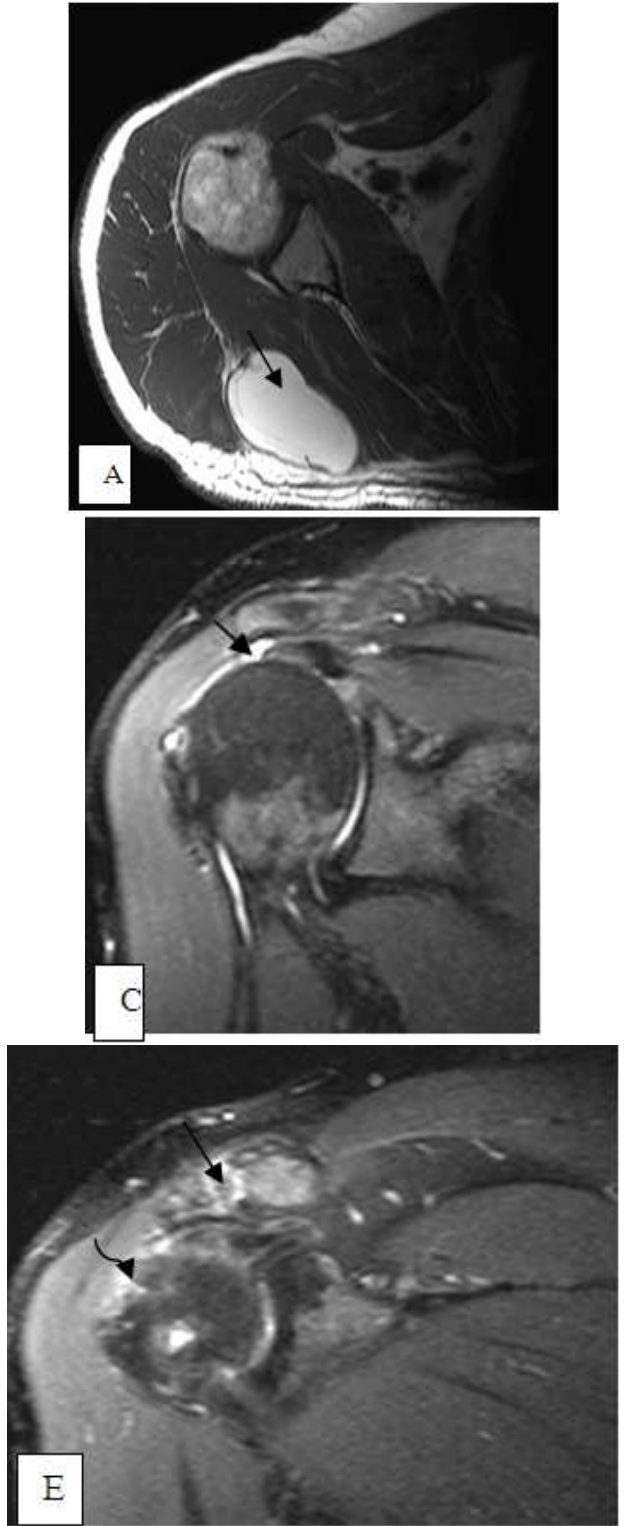
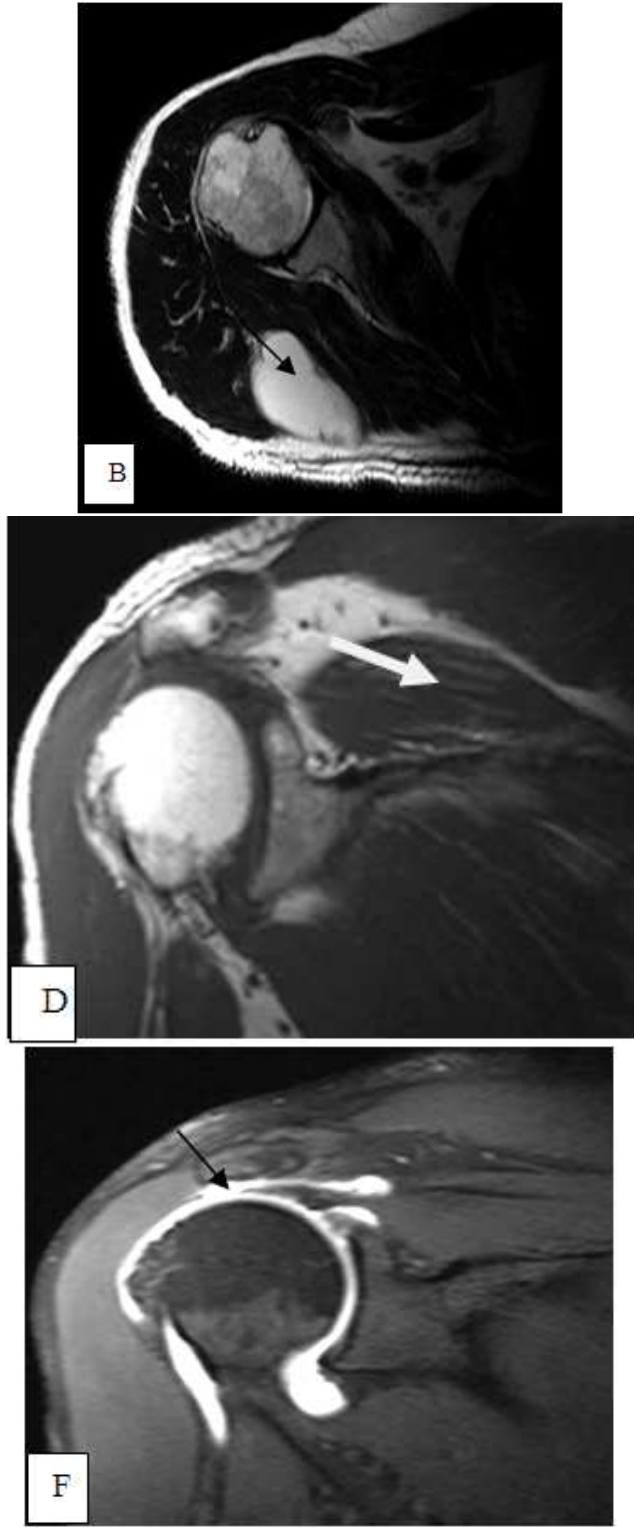

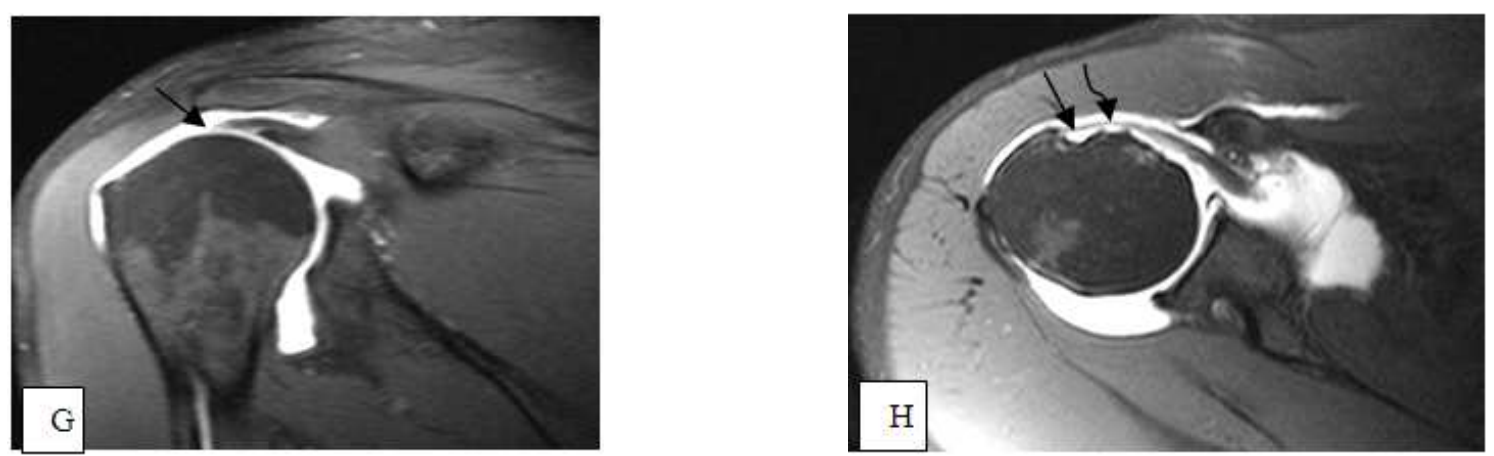

Fig. 5 (A-H). Male patient 53 years, complaining of shoulder pain and limitation of movement for about 10 years duration which became recently more severe. Image A (axial T1 WI) shows a well defined oval subcutaneous lesion compressing the lower aspect of the infraspinatus muscle showing hyperintense signal (arrow). Image B: (axial T2WI) shows a well defined oval subcutaneous lesion compressing the lower aspect of the infraspinatus muscle showing hyperintense signal (arrow). Image C (coronal oblique STIR) shows complete tear of the supraspinatus tendon with a gap measuring about $2 \mathrm{~cm}$ (arrow) filled with fluid and mild amount of joint effusion. Image D (coronal oblique T1WI) shows hyperintense streaks of fat signal in the supraspinatus muscle (arrow). Image E: (coronal oblique STIR) shows acromioclavicular joint osteoarthritis (arrow) and glenohumeral osteoarthritic changes with subchondral cystic changes (curved arrow).Image F: (coronal oblique T1WI with fat sat) post arthrography shows complete supraspintus tendon tear (arrow). Image G: (coronal oblique T1WI fat sat) post arthrography shows complete infraspinatus tendon tear (arrow). Image H: (axial T1WI fat sat) post arthrography shows empty bicipital grove (arrow) and partial tear of the subscapularis tendon (curved arrow).

\section{Discussion}

Magnetic resonance imaging is an efficient investigation in shoulder joint assessment. It has excellent resolution, soft tissue contrast and provides multiplanar imaging. It is a reliable technique for the evaluation of the rotator cuff tendons abnormality and offers excellent depiction of both soft tissue and osseous structures. MRI also has been used for the observation of extra-articular anatomy (lipoma in fig. $5 \mathrm{~A} \& \mathrm{~B})$, which an arthroscope cannot do (6).

Recent advances in MR imaging have contributed to shorter scanning times and higher-quality images. An understanding of imaging techniques, normal variants, technical artifacts, and diagnostic pitfalls will improve diagnostic accuracy of shoulder studies (7).

Arthroscopy is the only investigation that allows the direct visualization of the shoulder joint. It is considered by many as the best technique for diagnosing shoulder disorders and subsequently creating a treatment plan (8).

Seventy four of our patients were $\geq 31$ years old and twenty six patients were $<31$ years. Conventional MRI showed that patients below 31 years showed higher incidence of labral injuries while those $\geq 31$ years showed higher incidence of rotator cuff tendon injuries. This could be explained by the fact that most rotator cuff lesions are due to degenerative changes that increase with advancing age while labral lesions are mostly due to trauma, violence, and sport injuries or congenital in origin. The Rotator cuff tendon pathological lesions were the most common, involving mostly the supraspinatus tendon. Conventional MRI showed $76.9 \%$ sensitivity and $100 \%$ specificity in detection of partial supraspinatus tendon tears (fig. $1 \mathrm{C}$ ) and $83.3 \%$ sensitivity and $100 \%$ specificity in detection of full thickness supraspinatus tears (fig. 5 F). Thirty supraspinatus partial thickness tears and ten supraspinatus full thickness tears were diagnosed by conventional MRI while arthroscopy diagnosed 39 supraspinatus partial thickness tears and 12 full thickness tears.

This agrees with the study performed by Magee et al., (9) to report the diagnostic sensitivity of conventional MRI versus MR arthrography of the shoulder. One hundred fifty patients were examined by conventional MRI followed by MRA and the results of both techniques were compared with arthroscopy. Conventional MRI sensitivity was $92 \%$ for full-thickness supraspinatus tendon tears, $68 \%$ for articular surface partial-thickness supraspinatus tendon tears, and $84 \%$ for bursal surface supraspinatus tendon tears compared with arthroscopy. The higher sensitivity compared to our results may be because they used a 3T machine and the larger sample size (150 patients).

Our results were comparable to those of Zlatkin et al., (10). They examined 121 patients complaining of shoulder pain with clinically suspected rotator cuff tendon tear by conventional MRI $1.5 \mathrm{~T}$ machine. MRI sensitivity was $91 \%$ and specificity was $88 \%$ for detection of partial and full-thickness supraspinatus tendon tears compared with arthroscopy.

In the study by Wnorowski et al. (11), they examined 39 shoulders (38 patients) and compared the accuracy of MRI interpretation of rotator cuff integrity relative to arthroscopy. They found that routine MRI showed only $20 \%$ sensitivity in detecting partial-thickness tears and $71 \%$ for full thickness tears. They concluded that MRI was most helpful if the results were negative, and MRI diagnosis of partial tear was of little value. Considering the high cost of shoulder MRI, this study has significant implications for the evaluation of patients with possible rotator cuff pathology.

Our study showed $76.9 \%$ conventional MRI sensitivity and $100 \%$ specificity in detection of anterior labral tears (osseous Bankart fig. 4 A). The sensitivity was $54.5 \%$ and the specificity was $98.9 \%$ in detection of SLAP tears (SLAB type II, fig. 1 B)

In the study performed by Magee et al., (9) conventional MRI sensitivity was $83 \%$ for detection of anterior labral 
tears and $83 \%$ for detection of SLAP tears.

In our study the low sensitivity of conventional MRI $(54.5 \%)$ in detection of SLAP tears could be explained by the small sample size (11 patients).

Conventional MR imaging, allows direct visualization of major anatomic structures, yet its deficiency in depicting glenohumeral ligament lesions has led to the increasing use of arthrographic techniques (12).

MR Arthrography following conventional MRI was performed only to eight patients in this study. The rest of the patients refused the procedure because they knew it would be followed by arthroscopy and both were invasive procedures. The results obtained by MRA and arthroscopy were compared and were found identical. MRA diagnosed 1 Bankart lesion, 2 SLAP tears, 1 SS partial thickness tear, 1 full SS tear and 1 infraspinatus full thickness tear. Also MR Arthrography diagnosed 1 partial subscapularis tendon tear, 1 complete biceps tendon tear, 3 sublabral foramina and 4 sublabral recesses. One partial SS tendon tear, 1 SLAP tear, 1 complete infraspintus tendon tear, 1 partial subscapularis tendon tear, 1 torn IGHL, 1 absent MGHL and 1 sublabral recess were missed by conventional MRI but were diagnosed by MRA thus MRA improves the detection of different shoulder lesions compared to conventional MRI.

Jbara et al., (13) examined 87 patients complaining of shoulder pain in their study using $1.5 \mathrm{~T}$ machine. They showed $70 \%$ MRA sensitivity and $67 \%$ specificity in detecting anterior labral tears and $79 \%$ sensitivity and $85 \%$ specificity in detecting SLAP tears.

In the study performed by Magee et al., (9), three fullthickness supraspinatus tendon tears and nine partialthickness tears were seen on MRA but not on conventional MRI. MRA sensitivity was $100 \%$ for full-thickness supraspinatus tendon tears, $97 \%$ for partial-thickness articular surface supraspinatus tendon tears, and $84 \%$ for partial-thickness bursal surface supraspinatus tendon tears compared with arthroscopy .

Al Hiari (14) study included twenty eight patients who had history of previous shoulder dislocation and clinical suspicion of anteroinferior labroligamentous lesions and glenohumeral joint instability. All patients underwent shoulder MRA and the results were compared with the arthroscopic findings which were used as the reference standard. MRA results were analyzed for the presence and type of labroligamentous injuries. By arthroscopy, 21 anteroinferior labroligamentous lesions were diagnosed, including 15 Bankart lesions, three ALPSA lesions, two Perthes lesions and one GLAD lesion. When compared with arthroscopic findings, shoulder MRA had two falsenegative results (sensitivity, 92.8\%) and no false-positive results. The sensitivity of shoulder MRA in detecting anteroinferior labroligamentous lesions was 92.8\% (26/28) and specificity was $(100 \%)$. The overall accuracy of shoulder MRA in detecting labroligamentous lesions in this study was $90.5 \%$.

There were few limitations to our study the most important of which was fear of most of the patients from the interventional procedure of MRA, failure of adequate distension of the joint due to inconvenience of the patient during joint filling and the small number of patients having SLAP tears, one case of Perthes and absent other labral variant lesions which did not allow further statistical analysis.

\section{Conclusion}

MRI of the shoulder is an accurate, useful and established technique for assessment of rotator cuff pathology especially detecting full-thickness tears; however, it was found to be less reliable in detecting partial-thickness tears.

MRA is more accurate than MRI to clarify the intraarticular anatomy, intra-articular damage and the identification and differentiation of intra-articular pathologies such as the ALPSA lesion from a Bankart lesion, which would necessitate a different approach to the surgical repair. MRA appears to be less effective than arthroscopy as regards capsular laxity and identification of loose bodies.

With technologic advances allowing for faster imaging times, improved spatial resolution and tissue contrast enhancement can be achieved and allow excellent visualization of the labrum and so MRA will not be routinely used.

\section{Conflict of Interest}

The authors have no conflict of interest to declare.

\section{References}

[1] Ostler S. Imaging the shoulder. Imaging 2003; 15: 162-173.

[2] Lambert A, Loffroy R, Guiu B, et al. Rotator cuff tears: value of 3.0T MRI. J Radiol. 2009; 90(5 Pt 1):583-8.

[3] Liauger J, Palmer J, et al. Non septic monoarthritis: imaging features with clinical and histopathologic correlation. Radiographic 2000; 20: S 263-78.

[4] Ruotolo C and Nottage WM. Surgical and nonsurgical management of rotator cuff tears. Arthroscopy 2000; 18: $527-531$.

[5] Boenisch U, Lembcke O and Naumann T. Classification, clinical findings and operative treatment of degenerative and posttraumatic shoulder disease: what do we really need to know from an imaging report to establish a treatment strategy? Eur J Radiol 2000; 35: 103-118.

[6] Cerynik DL, Ewald TJ, Sastry A, et al. Outcomes of isolated glenoid labral injuries in professional baseball pitchers. Clin J Sport Med. 2008; may18 (3):255-8.

[7] Magee T, Williams D, Mani N. Shoulder MR arthrography: which patient group benefits most? AJR Am J Roentgenol 2004; 183: 969 -974. 
[8] Beltran J, Jbara M, Maimon R. Shoulder: labrum and bicipital tendon. Topics in J Magn Reson Imaging 2003 Feb; 14(1):35-49.

[9] Thomas Magee. 3-T MRI of the Shoulder: Is MR Arthrography Necessary? AJR Am J Roentgenol 2009; 192:86-9.

[10] Zlatkin MB, Iannotti JP, Roberts MC, et al. Rotator cuff tears: diagnostic performance of MR imaging. Radiology $2002 ; 172: 223-227$.

[11] Wnorowski DC, Levinsohn EM, Chamberlain BC, et al. Magnetic resonance Imaging assessment of the rotator cuff: is it really accurate? Arthroscopy 1997; 13:710-9.
[12] Farber JM, Buckwalter KA. Sports related injuries of the shoulder: instability. Radiol Clin North Am 2002; 40:235 249 .

[13] Jbara M, Marten P, Morcos M, et al. Shoulder MR Arthrography: How, Why, When Radiol Clin N Am 2005; 43: 683-692.

[14] Al Hiari A (2008): Shoulder Instability: The Role of MR Arthrography in diagnosing Antero-Inferior Labroligamentous lesions our experience at King Hussein Medical Center. PAK J MED 2008; 24: 16-11. 